ఠ ORIGINAL RESEARCH

\title{
miR-4262, low level of which predicts poor prognosis, targets proto-oncogene CDI63 to suppress cell proliferation and invasion in gastric cancer
}

This article was published in the following Dove Medical Press journal: OncoTargets and Therapy

\author{
Hongzhi Zhang \\ Huijuan Jiang \\ Huixiang Zhang \\ Juncai Liu \\ Xigang Hu \\ Lei Chen
}

Department of Radiotherapy, Huaihe Hospital of Henan University, Kaifeng 475000, Henan, China
Correspondence: Hongzhi Zhang Department of Radiotherapy, Huaihe Hospital of Henan University, No 8, Baobei Road, Kaifeng 475000, Henan, China

Tel/fax+86 37I 23906621

Email zhz_7607@I26.com
Background: miR-4262 was identified as a tumor promoter in several cancers, but its exact role in gastric carcinoma is still largely unknown.

Methods: The expression of miR-4262 was detected in gastric cancer tissues. Different concentrations of miR-4262 mimic and miR-4262 antagomir were respectively transfected into primary gastric carcinoma cells. After incubation for $72 \mathrm{~h}$, the overexpression efficiencies were confirmed by qPCR, cell proliferation was detected with the CCK-8 assay, cell apoptosis was detected by using the PI/Annexin V Cell Apoptosis Kit, and cell invasion was detected with the Transwell invasion assay. The molecular mechanisms underlying the action of miR-4262 in gastric carcinoma cells were also explored.

Results: In this study, we found that miR-4262 was significantly downregulated in gastric tissue from gastric cancer patients compared with that from the control group. Moreover, the level of miR-4262 was significantly lower in advanced gastric carcinoma. Additionally, lower level of miR-4262 was correlated with poorer prognosis and lower survival rate in gastric cancer patients. Then, different concentrations of miR-4262 mimic and miR-4262 antagomir were transfected into primary gastric carcinoma cells, respectively. The results showed that miR-4262 mimic suppressed proliferation and invasion and promoted cell apoptosis in a dose-dependent manner in gastric carcinoma cells. In contrast, miR-4262 antagomir increased proliferation and invasion and decreased cell apoptosis in a dose-dependent manner in gastric carcinoma cells. Furthermore, miR-4262 could directly target and suppress the expression of the proto-oncogene CD163.

Conclusion: Our findings indicate that lower level of miR-4262 predicts poorer prognosis in gastric patients, and miR-4262 can target proto-oncogene CD163 to suppress gastric cancer cell proliferation and invasion.

Keywords: miR-4262, gastric carcinoma, prognosis, proliferation and invasion, CD163

\section{Introduction}

Gastric cancer is the most common digestive tract malignant tumor worldwide, although the incidence of gastric cancer has declined slightly in recent years. ${ }^{1}$ In most eastern and developing countries, many recent reports showed that gastric cancer patients tend to be younger, because of changes in diet structure, increased working pressure and other pathological factors.,3 For gastric cancer, the prognosis is quite closely related to the stage of disease at diagnosis. Patients with early gastric cancer present with symptoms indistinguishable from benign peptic ulcer diseases, and improvement of the detection rate of early gastric cancer is still a great challenge. ${ }^{4}$ Therefore, it is 
urgent to develop reliable biomarkers and therapeutic targets for gastric carcinoma.

ncRNAs are now widely recognized as functional RNA transcripts that are not able to encode proteins. The work of ncRNA research is mainly divided into the following two aspects: to identify new ncRNAs on a large scale and to study the function of ncRNAs through various methods. Highthroughput sequencing analysis has revealed a large number of ncRNAs, but only a few of them are characterized. miRNAs are a category of ncRNAs, which are about 22-nucleotide single-stranded small ncRNAs processed from stem-loop precursors. They have been involved in various biological processes under physiological and pathological conditions through post-transcriptionally suppressing different functional genes. ${ }^{5}$ Many studies revealed that miRNAs regulate proliferation, apoptosis, invasion, migration, transformation, cell cycle, angiogenesis and drug resistance in diverse tumor tissues. ${ }^{6}$ In gastric cancer, many miRNAs have been identified as regulatory factors of tumor occurrence and progression, as well as biomarkers for diagnosis and prognosis. ${ }^{7}$ For example, increased levels of miR-200 family are shown to be associated with tumor initiation, multiple key current indicators of early gastric cancer including tumor invasion depth, differentiation, histology and stage and overall survival rate. ${ }^{8}$ Another typical example is that miRNA-584-3p was identified as an independent prognostic factor associated with favorable outcome of gastric cancer, and it inhibits gastric tumor growth and metastasis by repressing Yin Yang 1-facilitated matrix metalloproteinase 14 expression. ${ }^{9}$

miR-4262 was identified as a tumor promoter in several cancers, but its exact role in gastric carcinoma is still largely unknown. In this study, the level of miR-4262 was detected in 48 volunteers and 347 patients with gastric cancer, and the prognostic value of miR-4262 level was evaluated. Then, the role of miR-4262 in the proliferation, apoptosis and invasion was investigated in primary gastric cancer cells. Furthermore, we explored the potential mechanism by which miR-4262 regulated the behaviors of gastric cancer cells.

\section{Materials and methods}

\section{Patients and ethics statement}

Forty-eight volunteers (aging 53.8 \pm 15.2 years) and 347 gastric cancer patients (aged 54.4 \pm 15.5 years) were enrolled in this study. Gastric epithelial tissue was obtained by using a singleuse Endoscope Live Sampling Needle (DT-EN-W3; Detian Medical Devices Co., Ltd., Changzhou, China) from the volunteers and during curative resection from gastric cancer patients. The study was conducted in accordance with the Declaration of Helsinki and approved by the ethical committee of Huaihe
Hospital of Henan University (China), and all the subjects gave written informed consent to participate in the study.

\section{Cell culture and transfection}

Primary gastric carcinoma cells were isolated from six patients by the tissue mass culture method. The cells were cultured in DMEM/F12 medium (Thermo Fisher Scientific, Waltham, MA, USA) supplemented with 10\% FBS (Thermo Fisher Scientific) and 10 ng/mL EGF (Sigma-Aldrich Co., St Louis, MO, USA). The cells were incubated in a humidified incubator with an atmosphere of $95 \%$ air $-5 \% \mathrm{CO}_{2}$ at $37^{\circ} \mathrm{C}$, and the medium was changed every 3 days.

Oligo miR-4262 mimics, antagomirs and relative negative controls (NCs) were synthesized and confirmed effective by RiboBio Company (Guangzhou, China). For transfection, cells were seeded into six-well plates at the density of $105 / \mathrm{cm}^{2}$. On reaching $70 \%$ confluence, different concentrations of miR-4262 mimics, miR-4262 antagomirs and relative NCs were, respectively, transfected into the cells with Lipofectamine 3000 (Thermo Fisher Scientific) according to the manufacturer's instructions. The medium was changed after 24 hours, and after incubation for another 48 hours, the cultures were applied in the following assays.

\section{Cell proliferation, apoptosis and invasion analyses}

Cell proliferation was evaluated by the Cell Counting Kit-8 (Sigma-Aldrich Co.) assay that was carried out according to the manufacturer's instructions.

Cell apoptosis was detected by using the PI/Annexin V Cell Apoptosis Kit (Sigma-Aldrich Co.) according to the manufacturer's instructions.

Cell invasion was detected by the Transwell Cell Invasion assay. In brief, cells were collected by centrifugation and were seeded into the plate at $10^{5} /$ well. Transwell chambers (Corning Incorporated, Corning, NY, USA) pre-coated with 1:8 Matrigel (Sigma-Aldrich Co.) were filled with serum-free medium. Serum-containing medium was then added into each lower chamber, followed by incubation at $37^{\circ} \mathrm{C}$ for 12 hours. The cells that invaded through the membrane were fixed in $4 \%$ paraformaldehyde for 30 minutes and stained with $0.1 \%$ crystal violet for 30 minutes. Then, cells across the membrane were counted under a light microscope (Olympus Corporation, Tokyo, Japan).

\section{Real-time quantitative PCR (qPCR)}

Total RNA was isolated using TRIzol reagent (Thermo Fisher Scientific). Real-time qPCR reactions were carried out in a final volume of $25 \mu \mathrm{L}$ and run in $\mathrm{iQ}^{\mathrm{TM}} 5$ Multicolor Real-Time 
PCR Detection System (Bio-Rad Laboratories Inc., Hercules, CA, USA). The reactions were initially denatured at $95^{\circ} \mathrm{C}$ for 1 minute followed by 35 cycles of $95^{\circ} \mathrm{C}$ for 15 seconds and $60^{\circ} \mathrm{C}$ for 60 seconds. The data were calculated by the $2^{-\Delta \Delta \mathrm{Ct}}$ method. miR-4262 stem-loop primer and quantitative primers, as well as U6 RNA primers (internal reference), were designed and produced by RiboBio Company.

\section{$3^{\prime}$-UTR luciferase reporter assay}

The cDNA fragment corresponding to the 3'-UTR of cluster of differentiation 163 (CD163) mRNA and the mutated fragment were, respectively, produced by PCR and nest PCR, and then they were individually cloned into psiCHECK ${ }^{\mathrm{TM}}-2$ Vectors (Promega Corporation, Fitchburg, WI, USA) between the Xho I and Not I sites. The constructs were confirmed by sequencing. $10^{4}$ HEK293T cells/well were seeded in 96-well plates. After 24 hours, transfections were performed using Lipofectamine 3000 Transfection Reagent (Thermo Fisher Scientific). Hundred nanograms of constructs were individually cotransfected with either $30 \mathrm{nM}$ of miR-4262 mimic or NC mimic. Cells were harvested at 48 hours after transfection, and luciferase activity was measured using the DualGlo Luciferase Assay System (Promega Corporation).

\section{Western blotting}

Thirty micrograms of protein were separated by $12 \%$ SDS-PAGE and electrotransferred to PVDF membrane (EMD Millipore, Billerica, MA, USA) for immunoblotting analysis. The CD163 rabbit polyclonal antibody (1:400; Abcam) and $\beta$-actin murine monoclonal antibody (1:800; Abcam) were, respectively, used to incubate with the membrane at $4{ }^{\circ} \mathrm{C}$ overnight. $\beta$-Actin was used as the internal reference. After incubation with the appropriate HRP-conjugate secondary antibody, the abundance of the proteins was detected using a ChemiDoc XRS Imaging System (Bio-Rad Laboratories Inc.).

\section{Statistical analyses}

The correlation between miR-4262 and clinicopathological characteristic was analyzed by one-way ANOVA. The Kaplan-Meier curve was used to evaluate the correlation between miR-4262 and the overall survival rate. For in vitro experiments, data were obtained from at least three independent experiments in every assay. Values were expressed as mean \pm SEM. Multiple comparisons were assessed by one-way ANOVA followed by Dunnett's tests with SPSS 22.0 software. Difference between groups was considered statistically significant if $P<0.05$.

\section{Results}

miR-4262 was downregulated in advanced gastric carcinoma

The level of miR-4262 was detected in the gastric epithelial tissue from 48 volunteers and 347 patients with gastric cancer by real-time qPCR. The results showed that miR-4262 significantly decreased in the gastric epithelial tissue of the gastric cancer patients (Figure 1A). Then, we, respectively, compared the level of miR-4262 in early and relatively late stages of gastric carcinomas. The results showed that the miR-4262 level was significantly lower in poorly differentiated tumor tissue compared with well and moderately differentiated tumor tissue (Figure 1B), lower in tumor tissue from patients at $\mathrm{T}_{2}-\mathrm{T}_{4}$ stages compared with that of $\mathrm{T}_{1}$ stage (Figure 1C) and lower in tumor tissue from patients at I/II stages compared with that of III/IV stages (Figure 1D). These results revealed that miR-4262 was preferentially expressed in early gastric carcinoma tissues. Then, to evaluate the prognostic value of miR-4262 in gastric cancer patients, the association between miR-4262 level and clinicopathological characteristics was analyzed, including age, gender, histologic grade, depth of invasion, lymph node metastasis, tumor metastasis and pathological stage. The results showed that there was no obvious association between miR-4262 level and age or gender of the patients (Table 1), while low level of miR-4262 was positively related to poor histologic grade, depth of invasion, lymph node metastasis, tumor metastasis and pathological stage (Table 1). The association between miR-4262 level and overall survival of patients was evaluated. Our results showed that patients with low level of miR-4262 displayed a significantly lower survival rate (Figure 1E). These abovementioned data indicated that the low level of miR-4262 predicts poor prognosis and low survival rate in gastric cancer patients.

\section{miR-4262 suppressed the proliferation and invasion in primary human gastric carcinoma cells}

Then, to explore the role of miR-4262 in the progression of gastric carcinoma, different concentrations of miR-4262 mimic were, respectively, transfected into primary gastric carcinoma cells. After incubation for 72 hours, the overexpression efficiencies were confirmed by qPCR (Figure 2A). Then, cell proliferation, apoptosis and invasion were detected. The results showed that miR-4262 mimic suppressed cell proliferation and invasion in a dose-dependent manner (Figure 2B and D). Moreover, miR-4262 mimic increased cell apoptosis in a dose-dependent manner (Figure 2C). Then, 
A

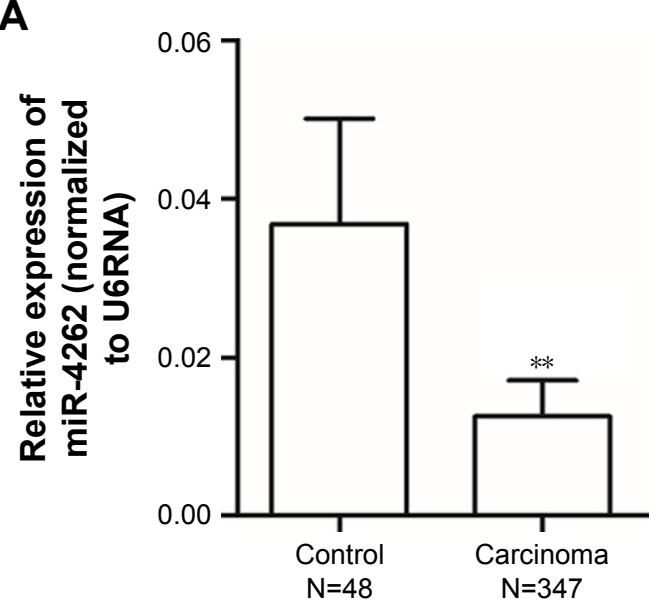

C

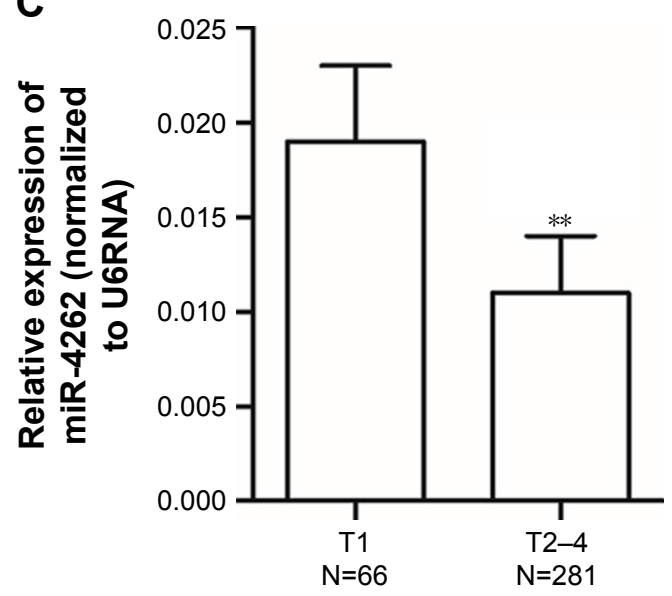

B

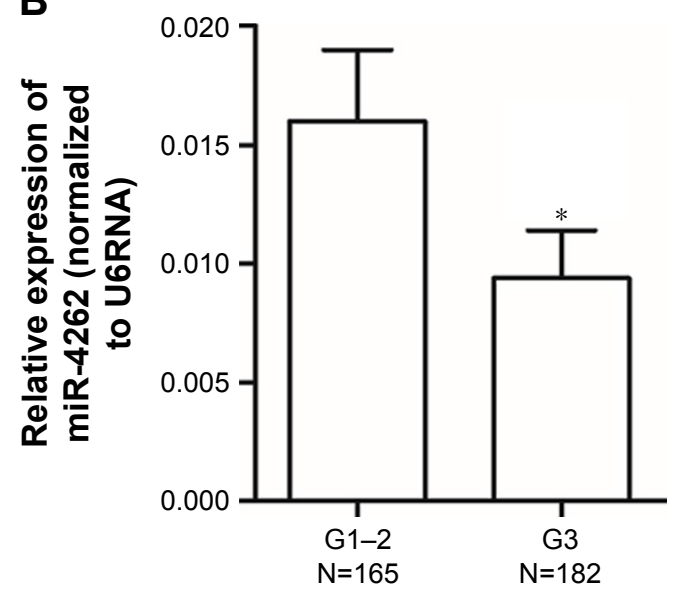

D

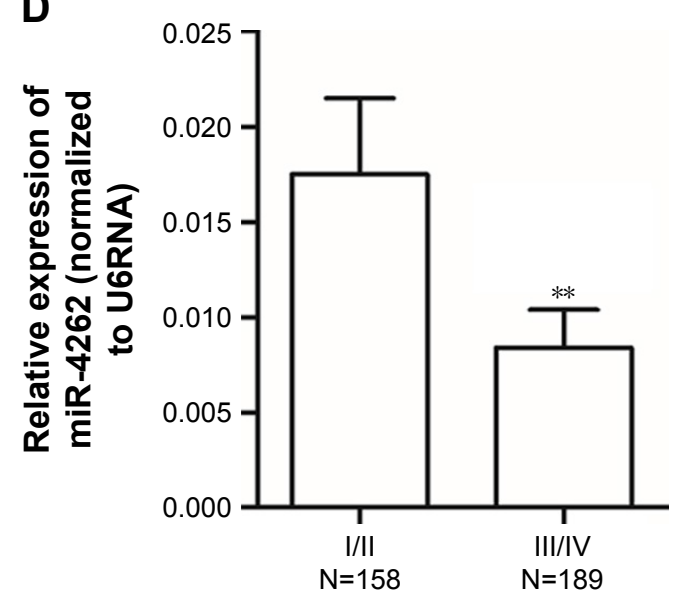

E

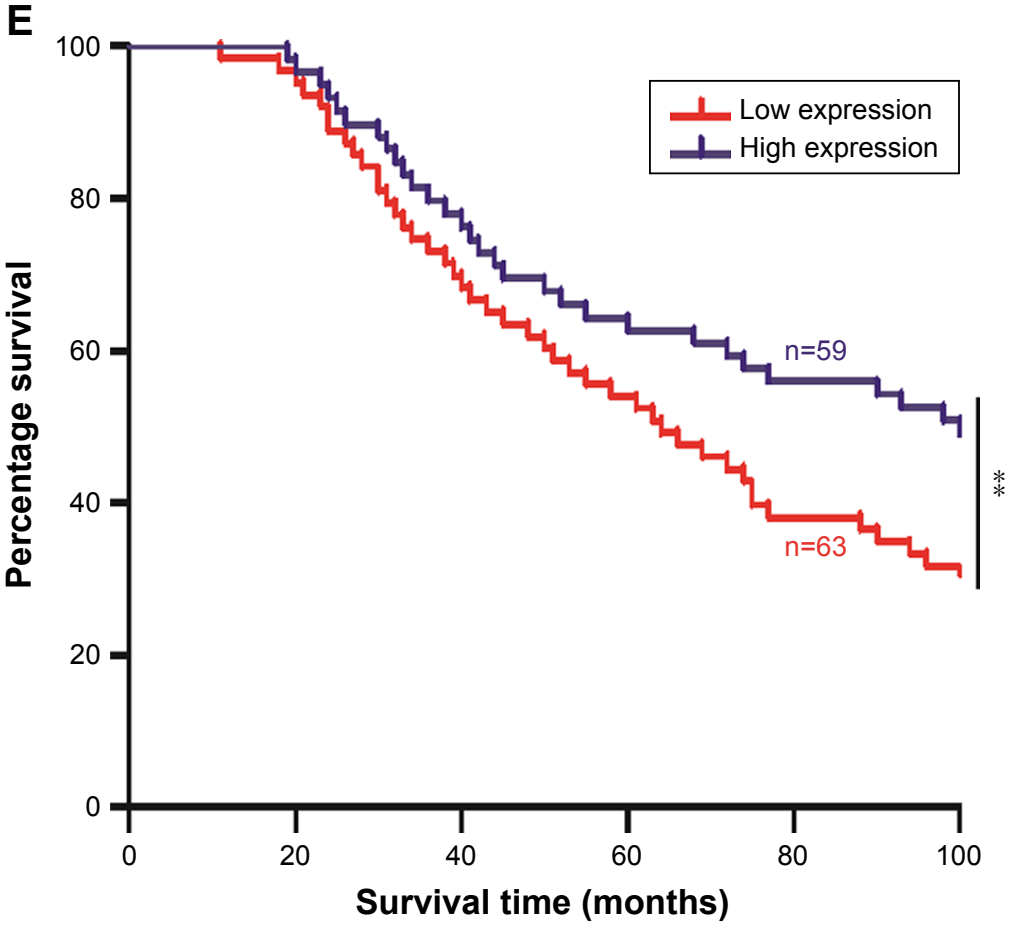

Figure I miR-4262 was significantly downregulated in advanced human gastric carcinoma.

Notes: (A) The expression of miR-4262 was detected in 48 volunteers and 347 patients with gastric cancer. The expression of CDI63 in different histologic grade (B), depth of invasion (C) and TNM stage (D). $* P<0.05$ and $* * p<0.01$. (E) The overall survival of patients with low level of miR-4262 ( $\leq 0.01, n=63)$ and high level $(\geq 0.02, n=59)$ was evaluated with the Kaplan-Meier survival curve. $* * P<0.0$ I. 
Table I Correlation between miR-4262 and clinicopathological characteristics in gastric cancer patients

\begin{tabular}{|c|c|c|c|}
\hline Indexes & Number of patients & Relative expression & $P$-value \\
\hline Age, years & & & 0.793 \\
\hline$<60$ & 135 & $0.0132 \pm 0.0031$ & \\
\hline$\geq 60$ & 212 & $0.0121 \pm 0.0032$ & \\
\hline Gender & & & 0.251 \\
\hline Male & 197 & $0.0122 \pm 0.0029$ & \\
\hline Female & 150 & $0.0130 \pm 0.0034$ & \\
\hline Histologic grade & & & $0.0039 * *$ \\
\hline Well and moderate & 165 & $0.0160 \pm 0.0037$ & \\
\hline Poor & 182 & $0.0094 \pm 0.0028$ & \\
\hline Depth of invasion & & & $0.0065^{* *}$ \\
\hline $\mathrm{T}_{1}$ & 66 & $0.0190 \pm 0.0040$ & \\
\hline $\mathrm{T}_{2}-\mathrm{T}_{4}$ & 281 & $0.0110 \pm 0.0030$ & \\
\hline Lymph node status & & & $0.0052 * *$ \\
\hline $\mathrm{N}_{0}$ & 101 & $0.0178 \pm 0.0035$ & \\
\hline $\mathrm{N}_{1}-\mathrm{N}_{3}$ & 246 & $0.0104 \pm 0.0032$ & \\
\hline Tumor metastasis & & & $0.0127^{*}$ \\
\hline$M_{0}$ & 282 & $0.0136 \pm 0.0025$ & \\
\hline$M_{1}$ & 65 & $0.0079 \pm 0.0026$ & \\
\hline Pathological stage & & & $0.0016 * *$ \\
\hline I and II & 158 & $0.0175 \pm 0.0036$ & \\
\hline III and IV & 189 & $0.0084 \pm 0.0021$ & \\
\hline
\end{tabular}

Notes: Measurements were expressed as mean \pm SD. One-way ANOVA was used for analysis, $* P<0.05$ and $* * P<0.0$ I.
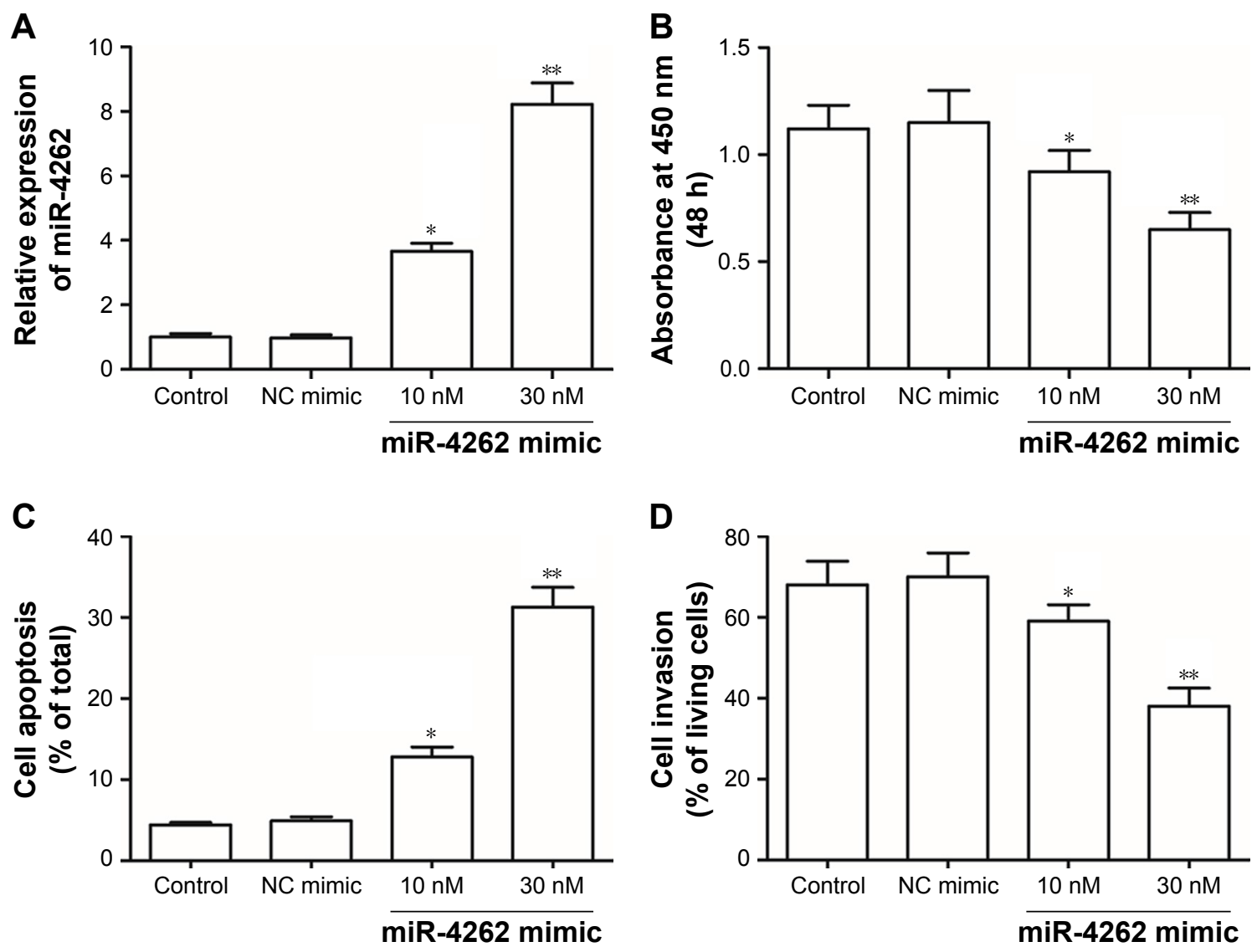

Figure 2 miR-4262 overexpression decreased the proliferation and invasion of primary gastric carcinoma cells in a dose-dependent manner.

Notes: (A) The overexpression efficiencies of different concentrations of miR-4262 mimic confirmed by qPCR. (B and C) miR-4262 mimic suppressed proliferation and increased apoptosis of gastric carcinoma cells in a dose-dependent manner. (D) miR-4262 mimic suppressed invasion of gastric carcinoma cells in a dose-dependent manner. Primary gastric carcinoma cells were isolated from six gastric cancer patients. Different concentrations of miR-4262 mimic were, respectively, transfected into primary gastric carcinoma cells. After incubation for 72 hours, the overexpression efficiencies were confirmed by qPCR, cell proliferation was detected by the CCK-8 assay, cell apoptosis was detected by using the $\mathrm{PI} /$ Annexin $\mathrm{V}$ Cell Apoptosis Kit, and cell invasion was detected by the Transwell invasion assay. $* P<0.05$ and $* * P<0.0 \mathrm{I}$ vs control.

Abbreviations: CCK-8, Cell Counting Kit-8; h, hours; NC, negative control; qPCR, quantitative PCR. 
A

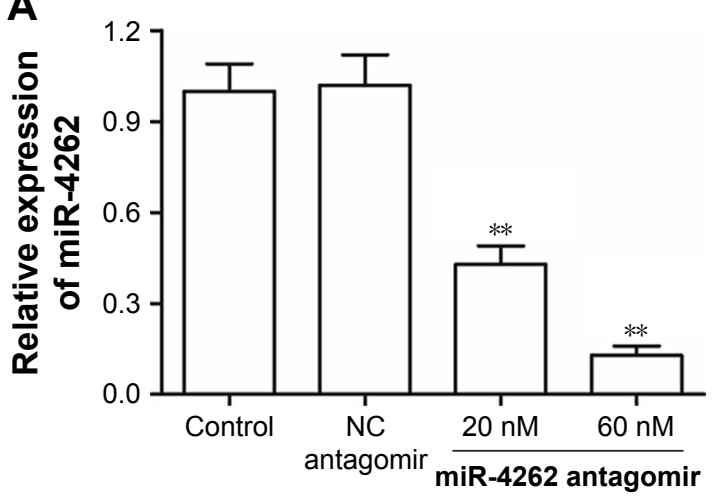

C

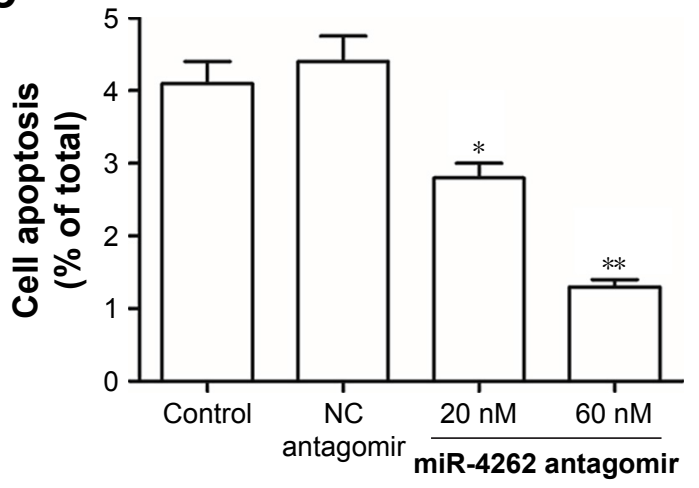

B

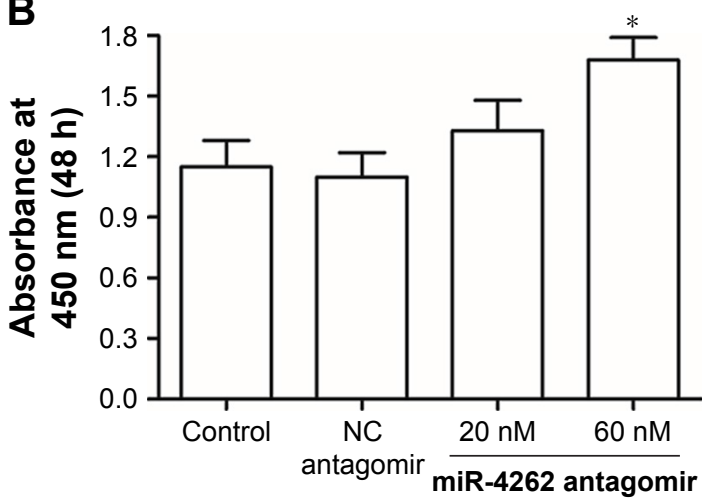

D

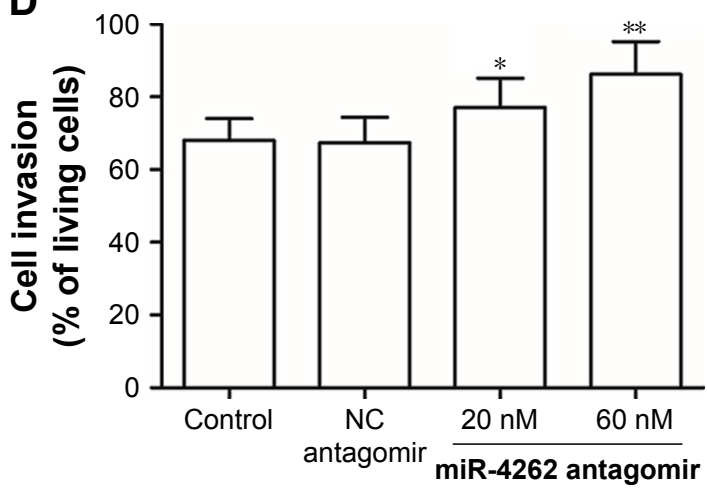

Figure 3 Knockdown of miR-4262 increased the proliferation and invasion of primary gastric carcinoma cells in a dose-dependent manner.

Notes: (A) The knockdown efficiencies of different concentrations of miR-4262 antagomir confirmed by qPCR. (B and C) miR-4262 antagomir promoted proliferation and decreased apoptosis of gastric carcinoma cells in a dose-dependent manner. (D) miR-4262 antagomir increased invasion of gastric carcinoma cells in a dose-dependent manner. Primary gastric carcinoma cells were isolated from six gastric cancer patients. Different concentrations of miR-4262 antagomir were, respectively, transfected into primary gastric carcinoma cells. After incubation for 72 hours, the knockdown efficiencies were confirmed by qPCR, cell proliferation was detected by the CCK-8 assay, cell apoptosis was detected by using the $\mathrm{Pl} / \mathrm{Annexin} \mathrm{V}$ Cell Apoptosis Kit, and cell invasion was detected by the Transwell invasion assay. $* P<0.05$ and $* * P<0.0 \mathrm{I}$ vs control. Abbreviations: CCK-8, Cell Counting Kit-8; h, hours; NC, negative control; qPCR, quantitative PCR.

different concentrations of miR-4262 antagomir were, respectively, transfected into primary gastric carcinoma cells. After incubation for 72 hours, the knockdown efficiencies were confirmed by qPCR (Figure 3A). In contrast to the results from miR-4262 mimic transfection, miR-4262 antagomir promoted cell proliferation and invasion and suppressed cell apoptosis in a dose-dependent manner (Figure 3B-D). These data demonstrated that miR-4262 functioned as a negative regulator in gastric carcinoma cell proliferation and invasion.

\section{miR-4262 directly targeted and negatively regulated expression of proto-oncogene CDI63}

Finally, the mechanism by which miR-4262 functioned as a negative regulator in gastric carcinoma cell proliferation and invasion was investigated. We searched the potential target genes using the bioinformatics tool. The results showed that the seed sequence of miR-4262 completely matched with seven continuous bases at the 3 '-UTR of CD163 mRNA (Figure 4A). A wild type (WT) CD163-3'-UTR and a mutated (MUT) luciferase reporter vector were, respectively, constructed and then applied in the following luciferase reporter gene assay. The detailed information for the mutated site is shown in Figure 4B. miR-4262 significantly suppressed the luciferase activity of WT CD163-3'-UTR luciferase reporter gene but could not change that of the MUT CD163-3'-UTR (Figure 4C). Then, Western blotting analysis showed that overexpression of miR-4262 dramatically decreased and knockdown of miR4262 increased the expression of CD163 protein (Figure 4D). These data demonstrated that miR-4262 directly targeted and negatively regulated the expression of proto-oncogene CD163.

\section{Discussion}

miR-4262 was discovered in human embryonic stem cells and neural lineages by Ago2-based immunoprecipitation in 2009. ${ }^{10}$ In this study, we found that the low level of miR-4262 was correlated with poor prognosis and low survival rate in gastric cancer patients. Gain- and loss-of-function experiments demonstrated that miR-4262 suppressed gastric cancer cell proliferation and invasion and increased cell apoptosis. 
A CD163 3'-UTR 5'-UAAAAAUUAAGUUUGUGAAUGUG-3' miR-4262 3'-GUCCAUCAGACUUACAG-5'

B CD163 3'-UTR-WT UAAAAAUUAAGUUUGUGAAUGUG CD163 3'-UTR-MUT UAAAAAUUAAGUUUG CGCCCGUG
C

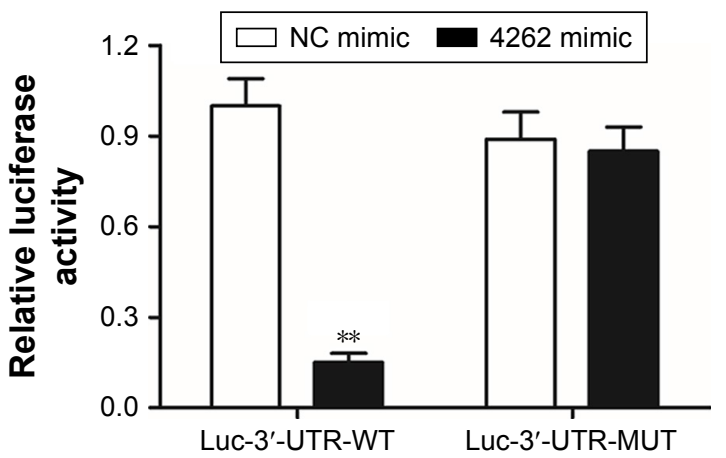

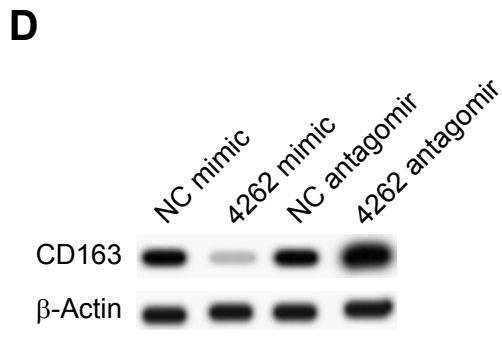

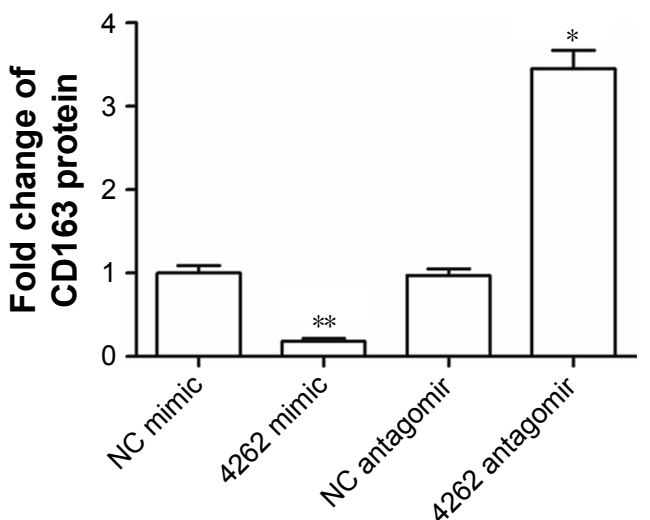

Figure 4 miR-4262 directly targeted and negatively regulated expression of proto-oncogene CDI63.

Notes: (A) miR-4262 potentially targeted CDI63 mRNA at the $3^{\prime}$-UTR. (B) The detailed information for the WT and MUT $3^{\prime}$-UTR luciferase reporter vector at the miR-4262 binding site. (C) Luciferase intensity detection in WT CD I63-3'-UTR luciferase reporter gene and the MUT CDI63-3'-UTR luciferase reporter gene. (D) miR-4262 negatively regulated expression of proto-oncogene CDI63. On reaching 70\% confluence, primary gastric carcinoma cells were transfected with $30 \mathrm{nM}$ miR-4262 mimics, $60 \mathrm{nM}$ miR-4262 antagomirs and $60 \mathrm{nM}$ relative NC oligos. After incubation for 72 hours, Western blotting was used to detect the expression of CDI63 protein. *P<0.05 and $* * P<0.01$ vs $N C$ mimic or NC antagomir.

Abbreviations: h, hours; MUT, mutated; NC, negative control; WT, wild type.

Our results suggested that miR-4262 functioned as a tumor suppressor in gastric cancer. As we know that the role of a miRNA was predominantly associated with the gene it targets, in contrast to most of the other cancer types, miR4262 was shown to play a promoting role in carcinogenesis. For example, the expression levels of miR-4262 were significantly upregulated in the bone marrow and serum of acute myeloid leukemia patients, and the high miR-4262 level predicted poorer overall survival time. ${ }^{11}$ In the cells of cutaneous malignant melanoma, breast cancer and hepatocellular carcinoma, miR-4262 promoted cell proliferation and invasion by activating the proto-onco pathway EGFR and NF- $\mathrm{KB} \cdot{ }^{12-14}$ However, in a couple of quite recent studies, miR-4262 was shown to target and transcriptionally suppress the expression of proto-oncogenes, osteopontin and Kaiso, ${ }^{15,16}$ which is similar to this study.

$\mathrm{CD163}$, with a molecular weight of $130 \mathrm{kDa}$, is the highaffinity scavenger receptor for the hemoglobin-haptoglobin complex found in 1987. ${ }^{17} \mathrm{CD} 163$ is famous as a marker of activated macrophage (M2 macrophage) in many immune disorders and inflammation-related diseases, such as obesity, chronic graft-vs-host disease, pulmonary tuberculosis and cardiovascular diseases. ${ }^{18-21}$ Recently, CD163 was identified as an important indicator for tumor-associated macrophages that were demonstrated to be strongly correlated with tumor aggressiveness in many types of cancers including gastric cancer. High expressions of CD163 in both macrophages and cancer cells are closely correlated with poor prognosis in gastric cancer. ${ }^{22-24}$ It was found that advanced gastric cancer stages coupled with higher expression of CD163 more frequently in recurrent patients, ${ }^{24,25}$ which implied that CD163 could be involved in gastric cancer progression and occurrence. Cheng et $\mathrm{a}^{26}$ demonstrated that knocking down CD163 in cancer cells could inhibit tumor growth in human gastric cancer cell-bearing BABL/c nude mice. In this study, we also found that downregulation of CD163 by miR-4262 displayed a suppressing role in gastric cancer cell proliferation and invasion.

STAT3 was regarded as a central regulatory factor for macrophage activation, which could be activated by many inflammatory factors and triggered by the differentiation of 
macrophage from M1 type to M2 type. ${ }^{27,28}$ CD163 was quite recently confirmed as a target gene of STAT3 in gastric cancer. ${ }^{26}$ Both soluble (in serum or cerebrospinal fluid) and insoluble (in the cell membrane the tumor cells or macrophages) CD163 now are identified as prognostic markers and potential therapeutic target for gastric cancer. CD163 was significantly upregulated in advanced gastric cancer, positively correlated with depth of invasion and pathological stage, and predicted poor overall survival in gastric cancer patients. ${ }^{26}$ In this study, we demonstrated that CD163 is a target gene of miR-4262 that displayed the opposite correlation with clinicopathological characteristics in gastric cancer: miR-4262 was downregulated in advanced gastric cancer; low level of miR-4262 was positively correlated with depth of invasion, lymph node status, tumor metastasis and pathological stage; and low miR-4262 lever predicted poor overall survival in gastric cancer patients.

There were two major limitations in this study. The one limitation is that owing to the lack of other detailed clinical indicators, we just compared the expression level of miR-4262 between gastric cancer patients at early and late stages. It would be helpful to evaluate the correlation between miR-4262 levels and clinical indicators of gastric cancer patients, such as tumor location, tumor size, depth of invasion, distal lymphatic metastasis, TNM stages and degree of cell differentiation. The other one is that the role of miR-4262 was just characterized in gastric cells in vitro. Results from tumor-bearing mice would be more convincing. In spite of these limitations, this study revealed that low levels of miR-4262 were associated with poor prognosis in gastric cancer patients and that miR-4262 negatively regulated proliferation and invasion of gastric carcinoma cells. Our findings suggested that miR-4262 might be regarded as a promising biomarker for gastric cancer prognosis, as well as a potential target for gastric cancer therapy.

\section{Conclusion}

miR-4262 was significantly downregulated in gastric cancer tissue, and lower level of miR-4262 was found in advanced gastric cancer. Low miR-4262 level was correlated with poor prognosis and low survival rate in gastric cancer patients. miR-4262 negatively regulated proliferation and invasion of gastric carcinoma cells via post-transcriptional suppression of proto-oncogene CD163.

\section{Disclosure}

The authors report no conflicts of interest in this work.

\section{References}

1. Lee SR, Kim HO, Yoo CH. Impact of chronologic age in the elderly with gastric cancer. J Korean Surg Soc. 2012;82(4):211-218.

2. Yan S, Bin LI, Jun-Qi WU, et al. Analysis of characteristics of clinical epidemiology about gastric cancer in hehuang valley. China Oncol. 2014;24(4):246-251.

3. de Martel C, Forman D, Plummer M. Gastric cancer: epidemiology and risk factors. Gastroenterol Clin North Am. 2013;42(2):219-240.

4. Dakubo JC, Clegg-Lamptey JN, Sowah P. Appropriateness of referrals for upper gastrointestinal endoscopy. West Afr J Med. 2011;30(5):342.

5. Carthew RW, Sontheimer EJ. Origins and mechanisms of miRNAs and siRNAs. Cell. 2009;136(4):642-655.

6. Zhong X, Coukos G, Zhang L. miRNAs in human cancer. J Pathol. 2015;223:102-115.

7. Wu HH, Lin WC, Tsai KW. Advances in molecular biomarkers for gastric cancer: miRNAs as emerging novel cancer markers. Expert Rev Mol Med. 2014;16:e1.

8. Song F, Yang D, Liu B, et al. Integrated microRNA network analyses identify a poor-prognosis subtype of gastric cancer characterized by the miR-200 family. Clin Cancer Res. 2014;20(4):878-889.

9. Zheng L, Chen Y, Ye L, et al. miRNA-584-3p inhibits gastric cancer progression by repressing Yin Yang 1-facilitated MMP-14 expression. Sci Rep. 2017;7(1):8967.

10. Goff LA, Davila J, Swerdel MR, et al. Ago2 immunoprecipitation identifies predicted microRNAs in human embryonic stem cells and neural precursors. PLoS One. 2009;4(9):e7192.

11. Han GT, Sun ZL, G.T. Han, Z.L. Up-regulation of serum miR-4262 predicts clinical outcome of patients with acute myeloid leukemia. Eur Rev Med Pharmacol Sci. 2017;21(9):2172.

12. Zhang D, Li Z, Zhang Y, Tu C, Huo J, Liu Y. miR-4262 promotes the proliferation of human cutaneous malignant melanoma cells through KLF6-mediated EGFR inactivation and p21 upregulation. Oncol Rep. 2016;36(6):3657-3663.

13. Wang K, Ren Y, Liu Y, Zhang J, He JJ. miR-4262 promotes proliferation and invasion of human breast cancer cells through directly targeting KLF6 and KLF15. Oncol Res. 2017;25(2):277-283.

14. Lu S, Wu J, Gao Y, Han G, Ding W, Huang X. MicroRNA-4262 activates the NF- $\kappa \mathrm{B}$ and enhances the proliferation of hepatocellular carcinoma cells. Int J Biol Macromol. 2016;86:43-49.

15. Song K, Liu N, Yang Y, Qiu X, Ning L, Xue Q. Regulation of osteosarcoma cell invasion through osteopontin modification by miR-4262. Tumour Biol. 2016;37(5):6493-6499.

16. Feng J. Upregulation of microRNA-4262 targets Kaiso (ZBTB33) to inhibit the proliferation and EMT of cervical cancer cells. Oncol Res. 2017;26(8):1215-1225.

17. Onofre G, Kolácková M, Jankovicová K, Krejsek J. Scavenger receptor CD163 and its biological functions. Acta Medica (Hradec Kralove). 2009;52(2):57-61.

18. Cinkajzlová A, Lacinová Z, Kloučková J, et al. An alternatively activated macrophage marker CD163 in severely obese patients: the influence of very low-calorie diet and bariatric surgery. Physiol Res. 2017;66(4):641-652.

19. Inamoto Y, Martin PJ, Paczesny S, et al. Association of plasma CD163 concentration with de novo-onset chronic graft-versus-host disease. Biol Blood Marrow Transplant. 2017;23(8):1250-1256.

20. Suzuki Y, Shirai M, Asada K, et al. Utility of macrophage-activated marker CD163 for diagnosis and prognosis in pulmonary tuberculosis. Ann Am Thorac Soc. 2017;14(1):57-64.

21. Castley A, Williams L, James I, Guelfi G, Berry C, Nolan D. Plasma CXCL10, sCD163 and sCD14 levels have distinct associations with antiretroviral treatment and cardiovascular disease risk factors. PLoS One. 2016;11(6):e0158169.

22. Huang X, Pan Y, Ma J, et al. Prognostic significance of the infiltration of $\mathrm{CD} 163^{+}$macrophages combined with $\mathrm{CD} 66 \mathrm{~b}^{+}$neutrophils in gastric cancer. Cancer Med. 2018;7(5):1731-1741. 
23. Zhang WJ, Zhou ZH, Guo M, et al. High infiltration of polarized CD163+ tumor-associated macrophages correlates with aberrant expressions of CSCs markers, and predicts prognosis in patients with recurrent gastric cancer. J Cancer. 2017;8(3):363-370.

24. Park JY, Sung JY, Lee J, et al. Polarized CD163+ tumor-associated macrophages are associated with increased angiogenesis and CXCL12 expression in gastric cancer. Clin Res Hepatol Gastroenterol. 2016;40(3):357-365.

25. Maniecki MB, Etzerodt A, Ulhøi BP, et al. Tumor-promoting macrophages induce the expression of the macrophage-specific receptor CD163 in malignant cells. Int J Cancer. 2012;131(10):2320-2331.
26. Cheng Z, Zhang D, Gong B, Wang P, Liu F. CD163 as a novel target gene of STAT3 is a potential therapeutic target for gastric cancer. Oncotarget. 2017;8(50):87244-87262.

27. de Jonge WJ, van der Zanden EP, The FO, et al. Stimulation of the vagus nerve attenuates macrophage activation by activating the Jak2-STAT3 signaling pathway. Nat Immunol. 2005;6(8):844-851.

28. Takaishi K, Komohara Y, Tashiro H, et al. Involvement of M2-polarized macrophages in the ascites from advanced epithelial ovarian carcinoma in tumor progression via Stat3 activation. Cancer Sci. 2010;101(10) 2128-2136.

\section{Publish your work in this journal}

OncoTargets and Therapy is an international, peer-reviewed, open access journal focusing on the pathological basis of all cancers, potential targets for therapy and treatment protocols employed to improve the management of cancer patients. The journal also focuses on the impact of management programs and new therapeutic agents and protocols on

\section{Dovepress}

patient perspectives such as quality of life, adherence and satisfaction. The manuscript management system is completely online and includes a very quick and fair peer-review system, which is all easy to use. Visit http://www.dovepress.com/testimonials.php to read real quotes from published authors.

Submit your manuscript here: http://www.dovepress.com/oncotargets-and-therapy-journal 